\title{
The Estimation of Radial Exponential Random Vectors in Additive White Gaussian Noise
}

\author{
P. Kittisuwan' ${ }^{1}$, S. Marukatat ${ }^{2}$, W. Asdornwised ${ }^{1}$ \\ ${ }^{1}$ Department of Electrical Engineering, Faculty of Engineering, \\ Chulalongkorn University, Bangkok, Thailand \\ ${ }^{2}$ Image Laboratory, National Electronics and Computer Technology Center (NECTEC), Thailand \\ Email:pichidkit@yahoo.com, sanparith.marukatat@nectec.or.th,widhyakorn.a@chula.ac.th \\ Received April 22, 2009; revised June 15, 2009; accepted June 22, 2009
}

\begin{abstract}
Image signals are always disturbed by noise during their transmission, such as in mobile or network communication. The received image quality is significantly influenced by noise. Thus, image signal denoising is an indispensable step during image processing. As we all know, most commonly used methods of image denoising is Bayesian wavelet transform estimators. The Performance of various estimators, such as maximum a posteriori (MAP), or minimum mean square error (MMSE) is strongly dependent on correctness of the proposed model for original data distribution. Therefore, the selection of a proper model for distribution of wavelet coefficients is important in wavelet-based image denoising. This paper presents a new image denoising algorithm based on the modeling of wavelet coefficients in each subband with multivariate Radial Exponential probability density function (PDF) with local variances. Generally these multivariate extensions do not result in a closed form expression, and the solution requires numerical solutions. However, we drive a closed form MMSE shrinkage functions for a Radial Exponential random vectors in additive white Gaussian noise (AWGN). The estimator is motivated and tested on the problem of wavelet-based image denoising. In the last, proposed, the same idea is applied to the dual-tree complex wavelet transform (DT-CWT), This Transform is an over-complete wavelet transform.
\end{abstract}

Keywords: MMSE Estimator, Radial Exponential Random Vectors, Wavelet Transform, Image Denoising

\section{Introduction}

The denoising of a natural image corrupted by Gaussian noise is a classic problem in signal processing. The distortion of images by noise is common during its, acquisition, processing, compression, mobile and network transmission. Traditional algorithms perform image denoising based on threshold function methods, such as soft-threshold and hard-threshold [1]. If the wavelet transform and MMSE estimator are used for this problem, the solution requires a priori knowledge about wavelet coefficients. Therefore, two problems arise: 1) what kinds of distributions represent the wavelet coefficients? 2) What is the corresponding estimator (shrinkage function)?

Figure 1 illustrates the histogram of photographic image and PDF plots. The PDF plots illustrate the marginal Radial Exponential PDF and Gaussian PDF. The histogram in Figure $\mathbf{1}$ is very symmetric with zero mean and the histogram of wavelet coefficients are more like marginal Radial Exponential PDF, it is more peaked and the tails are heavier, than the Gaussian distribution.

It is known that the amplitude of wavelet coefficients tend to propagate across scales. This parent-child relation is also underlined by the empirical joint histogram between parent and child coefficients as shown in [2]. In [3], they developed a multivariate spherically contoured Laplacian density that is similar to the Radial Exponential random vectors in its function form, but the marginal of Laplacian random vectors are not Radial Exponential density. Indeed, Radial Exponential random vectors specialize to Laplacian random vectors in the scalar case $(d=1)$. In this paper we focus on Radial Exponential random vectors with local variances to model these locality and persistence properties of wavelet coefficients. The rest of this paper is organized as follows. In Section 2, the basic idea of Bayesian denoising will be briefly described. Subsection 2.1 describes wavelet coefficients model, these models try to capture the dependencies between a coeffi- 


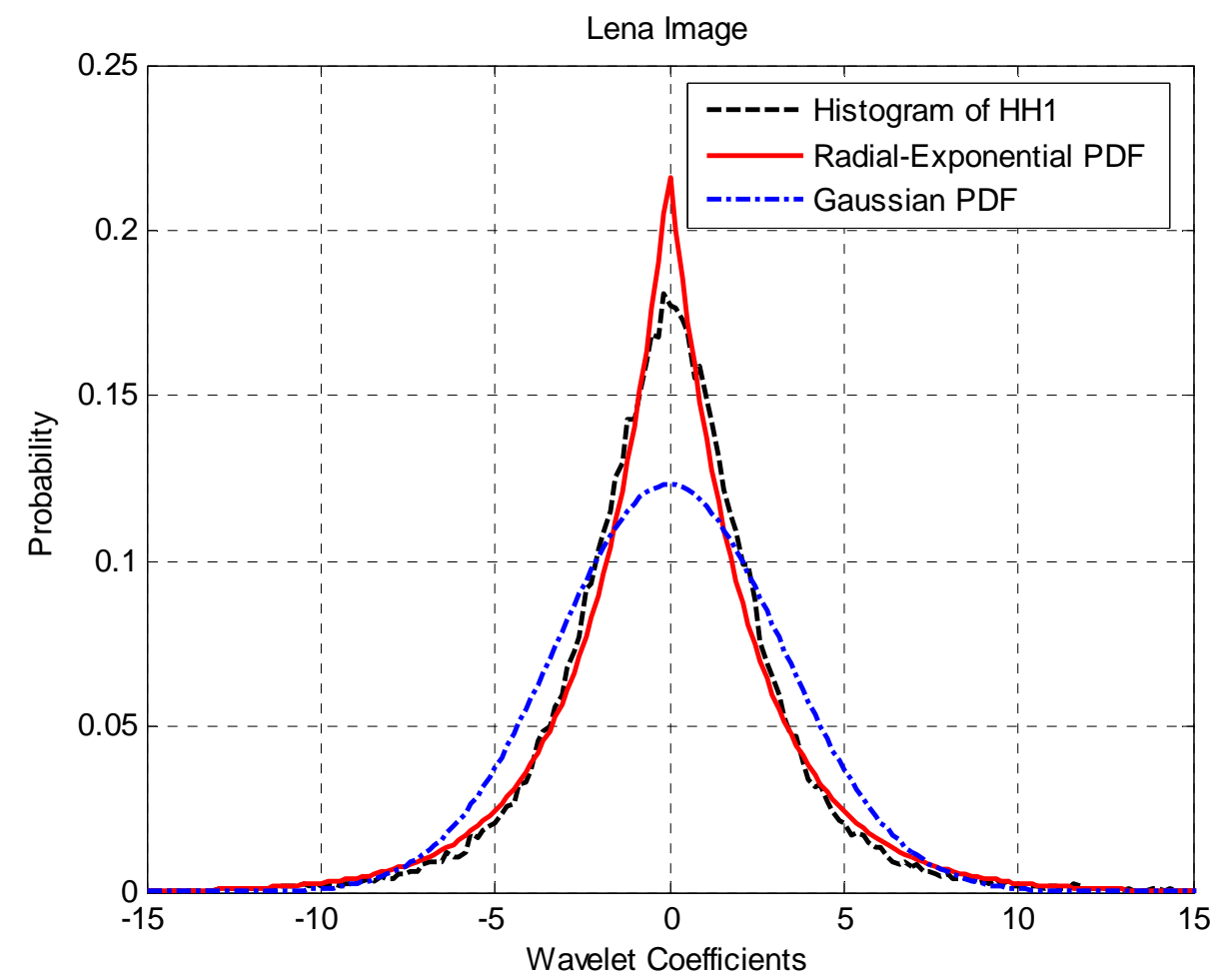

Figure 1. Histogram of wavelet coefficients in HH1 subband of $512 \times 512$ pixel Lena image.

cients and its group of parent in detail. In Section 3, we derive a closed form of MMSE estimator using multivariate Radial Exponential distribution with local variance, Radial-Shrink. Section 4 describes the approximated MAP (maximum a posteriori) estimation for local variances using Rayleigh density priori with Gaussian distribution (the local variances estimation of wavelet coefficients is the key to get better performance for image denoising). In Section 5, we use our model for wavelet based denoising of several images corrupted with additive Gaussian noise in various noise levels. The simulation results in comparison with MMSE TriShrink Laplace. In this paper, we not compare with BLS-GSM [4] because this method use a lot of time for denoising image. In the last simulation results, the performance of a subband dependent will be demonstrated on the dual-tree complex wavelet transform. The dual-tree complex wavelet transform (DT-CWT) [5,6] is an over-complete wavelet transform. The discrete wavelet transform (DWT) used in image denoising can be of many types, such as orthogonal/bi-orthogonal, real/ complex valued, separable/non-separable, or decimated/non-decimated. Due to the shift- invariance property, the over-complete trans-form improves the image denoising performance in PSNR by $1 \mathrm{~dB}$ as compared to that of the decimated representation [7]. Finally the concluding remarks are given in Section 6.

\section{Bayesian Denoising}

In this paper, we are interested in the problem of estimating d-component Radial Exponential random vectors, $\mathbf{x}$ in additive white Gaussian noise (AWGN), $\mathbf{n}$ The

$$
\mathbf{y}=\mathbf{x}+\mathbf{n}
$$

marginal models are weak models for wavelet coefficients of natural images because they ignore the dependencies between coefficients, although a coefficient and its parent are uncorrelated but are not independent. It is well known those wavelet coefficients are statistically dependent due to two properties of the wavelet transform 1) If a wavelet coefficient is large/small, the adjacent coefficients are likely to be large/small, and 2) large/small coefficients tend to propagate across the scales. Here, we can update the MMSE estimation problem as to take into account the statistical dependency between a coefficient and its group of parent. Let $x_{2}, x_{3}, \ldots, x_{d}$ represent the group of parent of $x_{1}\left(x_{2}, x_{3}, \ldots, x_{d}\right.$ is the wavelet coefficient at the same spatial position as $\mathrm{x}_{1}$, but at the next coarser scale). If we observe a noisy wavelet coefficient, $\mathbf{y}$ and $\mathbf{n}$ is additive Gaussian noise zero mean with variance $\sigma_{n}^{2}$, MMSE estimator can be rewritten as:

$$
\hat{x}_{1}(\mathbf{y})=\frac{1}{f_{\mathbf{y}}(\mathbf{y})} \int_{R^{d}} x_{1} f_{\mathbf{x}}(\mathbf{x}) f_{\mathbf{n}}(\mathbf{y}-\mathbf{x})|d \mathbf{x}|
$$




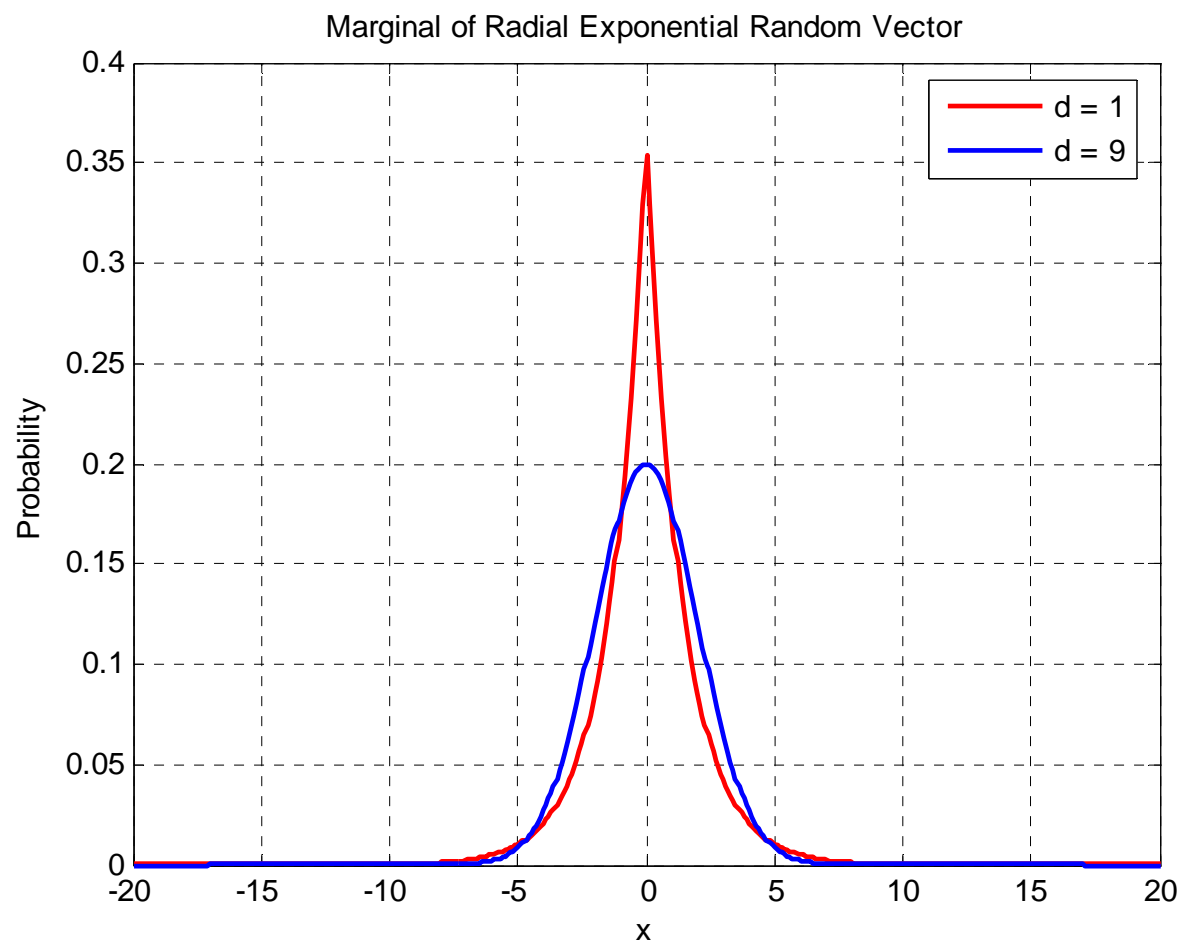

Figure 2. The marginal distribution of the d-component radial exponential PDF (3) for $d=1$ and $d=9, \sigma=4$, where $d=1$ the distribution is Laplacian PDF.

\subsection{Wavelet Coefficients Distribution}

In [3], and [8] a multivariate Laplacian distribution and bivariate Cauchy distribution are proposed to model wavelet coefficient and group of parent joint PDF. Figure 2 shows the marginal distribution of d-dimension Radial Exponential random vectors where $d=1$ and $d=9$. In case, $d=1$ (scalar) marginal distribution of Radial Expo-nential random vectors is Laplacian PDF. For $d>1$ the marginal distribution of Radial Exponential is less kurtosis than the Laplacian distribution. As d-dimension increases, the marginal distribution becomes more Gaussian. Indeed, the multivariate spherecally contoured of Radial Exponential distribution zero means with variance $\sigma^{2}$ has the density

$$
f_{\mathbf{x}}(\mathbf{x})=C \frac{1}{\sigma^{d}} \exp \left(-\frac{\sqrt{d+1}}{\sigma}\|\mathbf{x}\|\right), \mathbf{x} \in R^{d}
$$

$C$ is normalization constant parameter.

\section{MMSE Estimators with Radial Exponential Random Vectors}

\subsection{Generalized Incomplete Gamma Function}

In 1994, Chaudhry and Zubair introduced the generalize incomplete gamma function [3], defined as

$$
\Gamma(\alpha, x ; b)=\int_{x}^{\infty} t^{\alpha-1} \exp (-t-b / t) d t
$$

For $\alpha=\mathrm{Z}+1 / 2, Z$ are set of integer number, there is a closed from expression for the generalized incomplete gamma function, for $\alpha=1 / 2$ and $\alpha=-1 / 2$, for example, there is the formula

$$
\begin{aligned}
& \Gamma(1 / 2, x ; b)=0.5 \sqrt{\pi} \exp (-x)[\exp (-b / x) \\
& x \operatorname{erfcx}(\sqrt{x}-\sqrt{b / x})+\exp (-b / x) \operatorname{erfcx}(\sqrt{x}+\sqrt{b / x})], \\
& \Gamma(-1 / 2, x ; b)=0.5 \sqrt{\pi / b} \exp (-x)[\exp (-b / x) \\
& x \operatorname{erfcx}(\sqrt{x}-\sqrt{b / x})-\exp (-b / x) \operatorname{erfcx}(\sqrt{x}+\sqrt{b / x})]
\end{aligned}
$$

Here, numerical issues sometimes arise if some care is not taken. For example, computing the product of the first term in bracket of above equation can lead to numerical inaccuracies. However, note that $\exp (-b / x)$ $x \operatorname{erfcx}(\sqrt{x}-\sqrt{b / x})=\exp (x-2 \sqrt{b}) \operatorname{erfc}(\sqrt{x}-\sqrt{b / x})$. It can be determined which of the two expressions is the more accurate using following rule. If $x>2 \sqrt{b}$, then the first term in bracket is 
$\exp (-b / x) \operatorname{erfcx}(\sqrt{x}-\sqrt{b / x})$. Other cases, the first term in the bracket is $\exp (x-2 \sqrt{b}) \times \operatorname{erfc}(\sqrt{x}-\sqrt{b / x})$. Indeed, the generalized incomplete gamma function satisfies a recurrence relation for computing its values for other order, $\alpha$ from $[9,10]$

$$
\begin{aligned}
\Gamma(\alpha-1, x ; b)= & \frac{1}{b}[\Gamma(\alpha+1, x ; b)-\alpha \Gamma(\alpha, x ; b) \\
& \left.-x^{\alpha} \exp (-x-b / x)\right] .
\end{aligned}
$$

\subsection{MMSE Estimator with Multivariate Radial Exponential Distribution}

Multivariate spherically contoured of Radial Exponential density $\mathbf{x}$ can be generated by

$$
\mathbf{x}=\sqrt{\mathrm{z}} \mathbf{s},
$$

where $\mathbf{s}$ is d-component zero mean iid Gaussian random vectors with variance $\sigma^{2}$

$$
f_{\mathbf{s}}(\mathbf{s})=\frac{1}{\left(2 \pi \sigma^{2}\right)^{d / 2}} \exp \left(\frac{-\|\mathbf{s}\|^{2}}{2 \sigma^{2}}\right)
$$

and $z$ is a gamma PDF $f_{z}(z)=4 z \exp (-2 z), z>0$. The two distribution are iid. Setting $a=\sqrt{z}$, then $\mathbf{x}=a \mathbf{s}$. Changing the random variable of $(z, \mathbf{s}) \rightarrow(a, \mathbf{x})$. Using Jacobian transform $J=2 a\left(1 / a^{d}\right)$, then the PDF of random vectors $\mathbf{x}$ is given by

$$
\begin{aligned}
f_{\mathbf{x}}(\mathbf{x}) & =\int_{0}^{\infty}|J| f_{z}\left(a^{2}\right) f_{\mathrm{s}}\left(\frac{\mathbf{x}}{a}\right) d a \\
& =\int_{0}^{\infty} 2 a\left(\frac{1}{a^{d}}\right) f_{z}\left(a^{2}\right) f_{\mathrm{s}}\left(\frac{\mathbf{x}}{a}\right) d a
\end{aligned}
$$

From MMSE estimator (2), we would like to find $f_{\mathbf{y}}(\mathbf{y})$ and $A=\int_{R^{d}} f_{\mathbf{x}}(\mathbf{x}) f_{\mathbf{n}}(\mathbf{y}-\mathbf{x})|d \mathbf{x}|$. If the noise signal $\mathbf{n}$ is independent additive white Gaussian noise (AWGN) with variance $\sigma_{n}^{2}$,

$$
f_{\mathbf{n}}(\mathbf{n})=\frac{1}{\left(2 \pi \sigma_{n}^{2}\right)^{d / 2}} \exp \left(\frac{-\|\mathbf{n}\|^{2}}{2 \sigma_{n}^{2}}\right)
$$

First, the PDF of $\mathbf{y}$ is given by the multivariate convolution. The multivariate convolution defines as:

$$
f_{\mathbf{y}}(\mathbf{y})=\int_{R^{d}} f_{\mathbf{x}}(\mathbf{x}) f_{\mathbf{n}}(\mathbf{y}-\mathbf{x})|d \mathbf{x}|
$$

Using (6) gives

$$
\begin{aligned}
& f_{\mathbf{y}}(\mathbf{y})=\int_{R^{d}}\left[\int_{0}^{\infty} 2 a\left(\frac{1}{a^{d}}\right) f_{z}\left(a^{2}\right) f_{\mathrm{s}}\left(\frac{\mathbf{x}}{a}\right) d a\right] f_{\mathbf{n}}(\mathbf{y}-\mathbf{x})|d \mathbf{x}| \\
& =\int_{0}^{\infty} 2 a f_{z}\left(a^{2}\right)\left[\int_{R^{d}}\left(\frac{1}{a^{d}}\right) f_{\mathrm{s}}\left(\frac{\mathbf{x}}{a}\right) f_{\mathbf{n}}(\mathbf{y}-\mathbf{x})|d \mathbf{x}|\right] d a
\end{aligned}
$$

Using Gaussian convolution formula [3]

$$
\int_{R^{d}} \frac{1}{a^{d}} f_{\mathrm{s}}\left(\frac{\mathbf{x}}{a}\right) f_{\mathbf{n}}(\mathbf{y}-\mathbf{x})|d \mathbf{x}|=
$$

$$
\frac{1}{(2 \pi)^{d / 2}} \frac{1}{\left(a^{2} \sigma^{2}+\sigma_{n}^{2}\right)^{d / 2}} \exp \left(\frac{-\|\mathbf{y}\|^{2}}{2\left(a^{2} \sigma^{2}+\sigma_{n}^{2}\right)}\right)
$$

Therefore,

$$
\begin{aligned}
f_{\mathbf{y}}(\mathbf{y})= & \int_{0}^{\infty} 2 a f_{z}\left(a^{2}\right)\left[\frac{1}{(2 \pi)^{d / 2}} \frac{1}{\left(a^{2} \sigma^{2}+\sigma_{n}^{2}\right)^{d / 2}}\right. \\
& \left.\times \exp \left(\frac{-\|\mathbf{y}\|^{2}}{2\left(a^{2} \sigma^{2}+\sigma_{n}^{2}\right)}\right)\right] d a
\end{aligned}
$$

$$
\begin{aligned}
& =\int_{0}^{\infty} \frac{8 a^{3}}{(2 \pi)^{d / 2}\left(a^{2} \sigma^{2}+\sigma_{n}^{2}\right)^{d / 2}} \\
& \times \exp \left(-2 a^{2}-\frac{\|\mathbf{y}\|^{2}}{2\left(a^{2} \sigma^{2}+\sigma_{n}^{2}\right)}\right) d a
\end{aligned}
$$

Changing the variable of integration, using

$$
t=2 a^{2}+2 \sigma_{n}^{2} / \sigma^{2}, d t=4 a d a,
$$

gives

$$
\begin{gathered}
f_{\mathbf{y}}(\mathbf{y})=\frac{\exp \left(2 \sigma_{n}^{2} / \sigma^{2}\right)}{\left(\pi \sigma^{2}\right)^{d / 2}} \\
\times \int_{\frac{2 \sigma_{n}^{2}}{\sigma^{2}}}^{\infty} \frac{\left(t-2 \sigma_{n}^{2} / \sigma^{2}\right)}{t^{d / 2}} \exp \left(-t-\frac{\|\mathbf{y}\|^{2}}{\sigma^{2} t}\right) d t
\end{gathered}
$$

Using the generalized incomplete gamma function in (4), we get

$$
\begin{aligned}
f_{\mathbf{y}}(\mathbf{y})= & \frac{\exp \left(2 \sigma_{n}^{2} / \sigma^{2}\right)}{\left(\pi \sigma^{2}\right)^{d / 2}}\left[\Gamma\left(2-\frac{d}{2}, \frac{2 \sigma_{n}^{2}}{\sigma^{2}} ; \frac{\|\mathbf{y}\|^{2}}{\sigma^{2}}\right)\right. \\
& -\frac{2 \sigma_{n}^{2}}{\sigma^{2}} \Gamma\left(1-\frac{d}{2}, \frac{2 \sigma_{n}^{2}}{\sigma^{2}} ; \frac{\|\mathbf{y}\|^{2}}{\sigma^{2}}\right)
\end{aligned}
$$


Second, from MMSE estimator (2), we would like to find

$$
A=\int_{R^{d}} x_{i} f_{\mathbf{x}}(\mathbf{x}) f_{\mathbf{n}}(\mathbf{y}-\mathbf{x})|d \mathbf{x}|
$$

Using (6) gives

$$
\begin{aligned}
& A=\int_{R^{d}} x_{i}\left[\int_{0}^{\infty} 2 a\left(\frac{1}{a^{d}}\right) f_{z}\left(a^{2}\right) f_{\mathrm{s}}\left(\frac{\mathbf{x}}{a}\right) d a\right] f_{\mathbf{n}}(\mathbf{y}-\mathbf{x})|d \mathbf{x}| \\
& =\int_{0}^{\infty} 2 a f_{z}\left(a^{2}\right)\left[\int_{R^{d}} x_{i}\left(\frac{1}{a^{d}}\right) f_{\mathrm{s}}\left(\frac{\mathbf{x}}{a}\right) f_{\mathbf{n}}(\mathbf{y}-\mathbf{x})|d \mathbf{x}|\right] d a
\end{aligned}
$$

Using Gaussian convolution formula [3]

$$
\begin{gathered}
\int_{R^{d}} x_{i} \frac{1}{a^{d}} f_{\mathrm{s}}\left(\frac{\mathbf{x}}{a}\right) f_{\mathbf{n}}(\mathbf{y}-\mathbf{x})|d \mathbf{x}|= \\
\frac{y_{i} a^{2} \sigma^{2}}{\left(a^{2} \sigma^{2}+\sigma_{n}^{2}\right)} \frac{1}{\left(2 \pi\left(a^{2} \sigma^{2}+\sigma_{n}^{2}\right)\right)^{d / 2}} \exp \left(\frac{-\|\mathbf{y}\|^{2}}{2\left(a^{2} \sigma^{2}+\sigma_{n}^{2}\right)}\right)
\end{gathered}
$$

Therefore,

$$
\begin{aligned}
A=\int_{0}^{\infty} 2 a f_{z} & \left(a^{2}\right) \frac{y_{i} a^{2} \sigma^{2}}{\left(a^{2} \sigma^{2}+\sigma_{n}^{2}\right)} \frac{1}{\left(2 \pi\left(a^{2} \sigma^{2}+\sigma_{n}^{2}\right)\right)^{d / 2}} \\
& \times \exp \left(\frac{-\|\mathbf{y}\|^{2}}{2\left(a^{2} \sigma^{2}+\sigma_{n}^{2}\right)}\right) d a \\
& =\int_{0}^{\infty} \frac{8 a^{5} y_{i} \sigma^{2}}{(2 \pi)^{d / 2}\left(a^{2} \sigma^{2}+\sigma_{n}^{2}\right)^{d / 2+1}} \\
& \times \exp \left(-2 a^{2}-\frac{\|\mathbf{y}\|^{2}}{2\left(a^{2} \sigma^{2}+\sigma_{n}^{2}\right)}\right) d a
\end{aligned}
$$

Changing the variable of integration $a \rightarrow t$

$$
\begin{gathered}
A=\frac{4 \exp \left(2 \sigma_{n}^{2} / \sigma^{2}\right) y_{i}}{\left(\pi \sigma^{2}\right)^{d / 2}} \\
\times \int_{\frac{2 \sigma_{n}^{2}}{\sigma^{2}}}^{\infty} \frac{\left(t^{4} / 4-\left(\sigma_{n}^{2} / \sigma^{2}\right) t+\left(\sigma_{n}^{2} / \sigma^{2}\right)^{2}\right)}{t^{d / 2+1}} \exp \left(-t-\frac{\|\mathbf{y}\|^{2}}{\sigma^{2} t}\right) d t
\end{gathered}
$$

Using the generalized incomplete gamma function in (4), we get

$$
A=\frac{\exp \left(2 \sigma_{n}^{2} / \sigma^{2}\right) y_{i}}{\left(\pi \sigma^{2}\right)^{d / 2}}\left[\Gamma\left(2-\frac{d}{2}, \frac{2 \sigma_{n}^{2}}{\sigma^{2}} ; \frac{\|\mathbf{y}\|^{2}}{\sigma^{2}}\right)\right.
$$

$$
\left.-\frac{4 \sigma_{n}^{2}}{\sigma^{2}} \Gamma\left(1-\frac{d}{2}, \frac{2 \sigma_{n}^{2}}{\sigma^{2}} ; \frac{\|\mathbf{y}\|^{2}}{\sigma^{2}}\right)+4\left(\frac{\sigma_{n}^{2}}{\sigma^{2}}\right)^{2} \Gamma\left(-\frac{d}{2}, \frac{2 \sigma_{n}^{2}}{\sigma^{2}} ; \frac{\|\mathbf{y}\|^{2}}{\sigma^{2}}\right)\right]
$$

Solving (2) using (8) and (9) gives the MMSE estimator,

$$
\begin{aligned}
& \hat{x}_{i}=y_{i}\left[\Gamma\left(2-\frac{d}{2}, \frac{2 \sigma_{n}^{2}}{\sigma^{2}} ; \frac{\|\mathbf{y}\|^{2}}{\sigma^{2}}\right)\right. \\
& \frac{\left.-\frac{4 \sigma_{n}^{2}}{\sigma^{2}} \Gamma\left(1-\frac{d}{2}, \frac{2 \sigma_{n}^{2}}{\sigma^{2}} ; \frac{\|\mathbf{y}\|^{2}}{\sigma^{2}}\right)+4\left(\frac{\sigma_{n}^{2}}{\sigma^{2}}\right)^{2} \Gamma\left(-\frac{d}{2}, \frac{2 \sigma_{n}^{2}}{\sigma^{2}} ; \frac{\|\mathbf{y}\|^{2}}{\sigma^{2}}\right)\right]}{\left[\Gamma\left(2-\frac{d}{2}, \frac{2 \sigma_{n}^{2}}{\sigma^{2}} ; \frac{\|\mathbf{y}\|^{2}}{\sigma^{2}}\right)-\frac{2 \sigma_{n}^{2}}{\sigma^{2}} \Gamma\left(1-\frac{d}{2}, \frac{2 \sigma_{n}^{2}}{\sigma^{2}} ; \frac{\|\mathbf{y}\|^{2}}{\sigma^{2}}\right)\right]}
\end{aligned}
$$

Setting $d=3$ and using the recurrence relation of generalized incomplete gamma function (5), we get

$$
\begin{aligned}
& \hat{x}_{1}=y_{1}\left[\left(1+\frac{4\left(\sigma_{n}^{2}\right)^{2}}{\sigma^{2}\|\mathbf{y}\|^{2}}\right) \Gamma\left(\frac{1}{2}, \frac{2 \sigma_{n}^{2}}{\sigma^{2}} ; \frac{\|\mathbf{y}\|^{2}}{\sigma^{2}}\right)\right. \\
& +\left(\frac{2\left(\sigma_{n}^{2}\right)^{2}}{\sigma^{2}\|\mathbf{y}\|^{2}}-\frac{4 \sigma_{n}^{2}}{\sigma^{2}}\right) \Gamma\left(\frac{-1}{2}, \frac{2 \sigma_{n}^{2}}{\sigma^{2}} ; \frac{\|\mathbf{y}\|^{2}}{\sigma^{2}}\right) \\
& \left.-\frac{\left(2 \sigma_{n}^{2}\right)^{3 / 2}}{\left(\sigma^{2}\right)^{1 / 2}\|\mathbf{y}\|^{2}} \exp \left(-\frac{2 \sigma_{n}^{2}}{\sigma^{2}}-\frac{\|\mathbf{y}\|^{2}}{2 \sigma_{n}^{2}}\right)\right] \\
& \left.\left(\frac{1}{2}, \frac{2 \sigma_{n}^{2}}{\sigma^{2}} ; \frac{\|\mathbf{y}\|^{2}}{\sigma^{2}}\right)-\frac{2 \sigma_{n}^{2}}{\sigma^{2}} \Gamma\left(-\frac{1}{2}, \frac{2 \sigma_{n}^{2}}{\sigma^{2}} ; \frac{\|\mathbf{y}\|^{2}}{\sigma^{2}}\right)\right]
\end{aligned}
$$

We called this method Radial-Shrink.

\section{Parameter Estimation}

To apply our estimator, we need to know noise variance $\sigma_{n}^{2}$ and the variance of noise-free $\sigma^{2}$. To estimate noise variance from noisy wavelet coefficients, a robust median estimator is used from the $H H 1$ subband [11].

$$
\hat{\sigma}_{n}^{2}=\left(\frac{\operatorname{median}\left(\left|H H_{1}\right|\right)}{0.6745}\right)^{2}
$$

Under the assumption that marginal variance in wavelet child coefficient is difference for each data point 
$y(k)$, an estimated $\sigma^{2}(k)$ can be found using local neighborhood $N(k)$. We use a square window $N(k)$ centered at $y(k)$. To compute the variance of wavelet coefficients, we use the fact that the wavelet coefficients and the additive noise are independent, thus we have the following relation between their variance:

$$
\sigma^{2}(k)=\left(\sigma_{y}^{2}(k)-\sigma_{n}^{2}\right)_{+}
$$

where $\sigma_{y}^{2}(k)$ is the variance of noisy wavelet coefficient and $g_{+}=\max (0, g)$.

Now, assume that a prior marginal distribution $f_{\sigma_{y}^{2}(k)}\left(\sigma_{y}^{2}(k)\right)$ for each observed variance $\sigma_{y}^{2}(k)$ is available. Then we obtain an approximated MAP estimator for $\sigma_{y}^{2}(k)$ as [13]

$\sigma_{y}^{2}(k)=\underset{\sigma_{y}^{2}(k)}{\arg \max }\left[\ln \left(\prod_{j \in N(k)} f\left(y_{j} \mid \sigma_{y}^{2}(k)\right)\right) f_{\sigma_{y}^{2}(k)}\left(\sigma_{y}^{2}(k)\right)\right]$

In this paper, we assume $f\left(y_{j} \mid \sigma_{y}^{2}(k)\right)$, PDF of noisy wavelet coefficient, is Gaussian PDF $f\left(y_{j} \mid \sigma_{y}^{2}(k)\right)=\left(1 / \sqrt{2 \pi \sigma_{y}^{2}(k)}\right) \exp \left(-y_{j}^{2} /\left(2 \sigma_{y}^{2}(k)\right)\right)$ and $f_{\sigma_{y}^{2}(k)}\left(\sigma_{y}^{2}(k)\right)=\lambda_{1}^{2} \sigma_{y}^{2}(k) \exp \left(-\lambda_{1}\left(\sigma_{y}^{2}(k)\right)^{2} / 2\right), \lambda_{1}>0$, Rayleigh PDF. Using (13) and Cardanos' method [14] gives

$$
\sigma_{y}^{2}(k)=\sqrt[3]{C(k)}+\sqrt[3]{D(k)}
$$

where

$$
\begin{aligned}
& C(k)=\frac{\sum_{j \in N(k)} y_{j}^{2}}{4 \lambda_{1}^{2}}+\sqrt{\left(\frac{\left.\sum_{j \in N(k)} y_{j}^{2}\right)^{2}}{16 \lambda_{1}^{4}}+\frac{(M-2)^{3}}{216 \lambda_{1}^{6}}\right.} \\
& D(k)=\frac{\sum_{j \in N(k)} y_{j}^{2}}{4 \lambda_{1}^{2}}-\sqrt{\frac{\left(\sum_{j \in N(k)} y_{j}^{2}\right)^{2}}{16 \lambda_{1}^{4}}+\frac{(M-2)^{3}}{216 \lambda_{1}^{6}}}
\end{aligned}
$$

giving that $\lambda_{1}$ is parameter of Rayleigh PDF and $M$ is number of wavelet coefficient in $N(k)$.

To select the parameter $\lambda_{1}$, we use the fact that under our Rayleigh PDF prior assumption $\sigma_{y}^{2}(k)$, computed over all coefficients should distribute according to Rayleigh
PDF. First, the parameter $\lambda_{1}$ has calculated from the maximum likelihood estimation of $\sigma_{y}^{2}(k)$ that is

$$
\sigma_{y, M L}^{2}(k)=\sum_{j \in N(k)} y_{j}^{2} / M
$$

where [12]

$$
\lambda_{1}=\sqrt{\frac{2 N}{\sum_{k=1}^{N}\left(\sigma_{y, M L}^{2}(k)\right)^{2}}}
$$

where $N$ is number of wavelet coefficient in each subband. Using (12), we finally obtain the noise-free variance,

$$
\sigma^{2}(k)=\left((\sqrt[3]{C(k)}+\sqrt[3]{D(k)})-\sigma_{n}^{2}\right)_{+}
$$

\section{Experimental Results}

This section presents image denoising examples in wavelet domain to show efficiency of our new model and compare it with other method in literature. Due to space limitation, however, we give in this section results concerning two $512 \times 512$ grayscale images and one $256 \times 256$, namely, Lena, Boat, and Cameraman two types of wavelet representations, namely the decimated discrete wavelet transform (DWT) and dual-tree complex wavelet transform (DT-CWT). The images are obtained from USC-SIPI image database [7]. We also tested our algorithm using different additive Gaussian noise levels $\sigma_{n}^{2}=5,10,20,30$ and 40 and compared with MMSE_TriShrink_Laplace [3], Figure 3 shows the original cropped image Lena, its noisy version $\sigma_{n}^{2}=20$, and DWT-based denoising versions provide by two different methods, namely MMSE_ TriShrink_Laplace and Radial-Shrink The window size $7 \times 7$ are used. We have also investigated different window sizes. A $9 \times 9$ window size can also be a good choice. However, using $3 \times 3$ window size resulted in a slight performance loss. In this paper, we have not considered different square shapes for $N(k)$. Performance analysis is done using the PSNR measure. The results can be seen in Table 1-3. Each PSNR value in these tables is averaged over five runs. In these tables, the highest PSNR value is bolded.

For practical issue, an image, which is processed by our method, has some pixel values that are over the range of gray scale in MATLAB [1-256]. In this paper, we present the solution figuring out an average from the neighboring pixels and use the average value instead. 


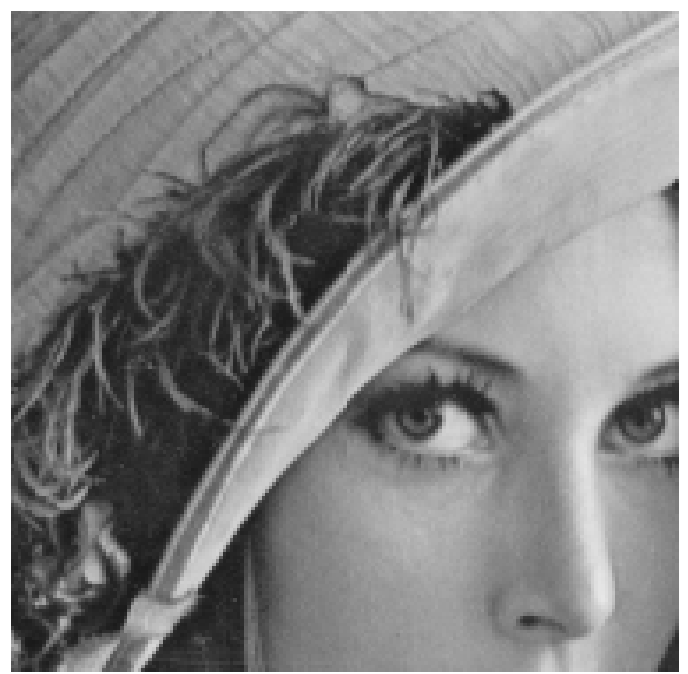

(a)

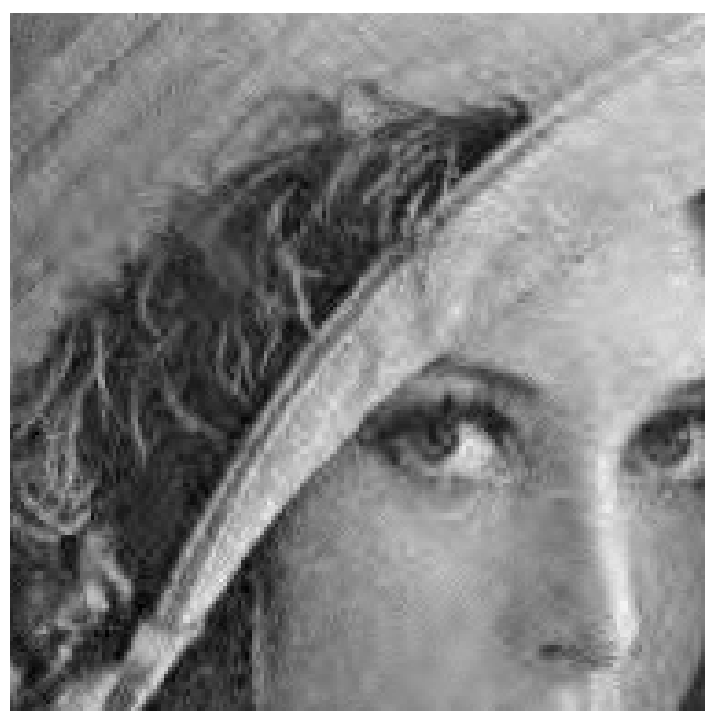

(a)

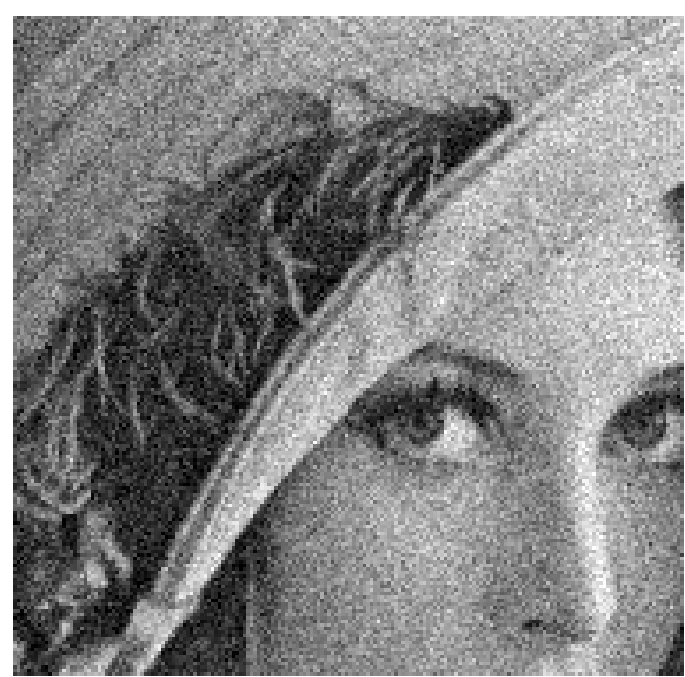

(b)

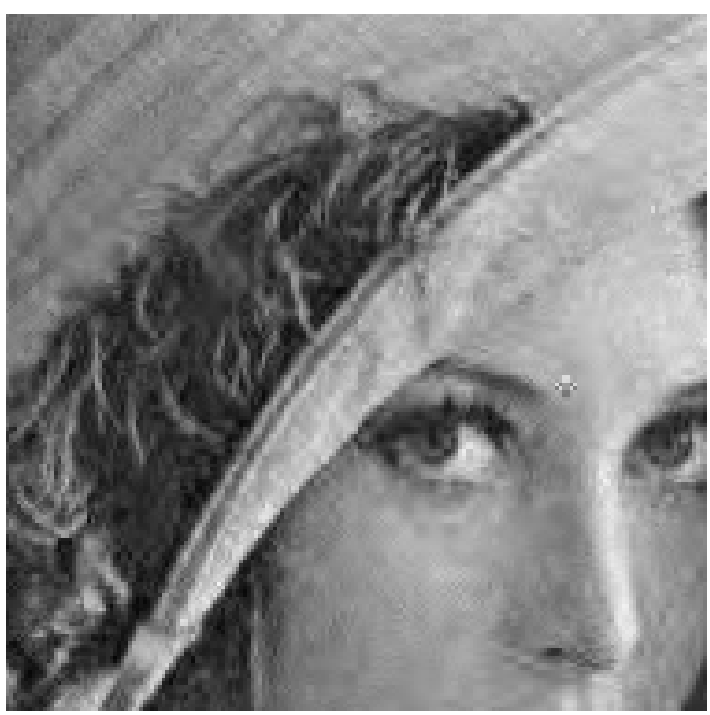

(b)

Figure 3. Comparison of the denoising images obtained from Bayesian estimator DWT-based denoising algorithms on Lena with $\sigma_{n}^{2}=20$ : (a) Noise-free image, (b) Noisy image, (c) MMSE_TriShrink_Laplace [3] (PSNR = 30.15), and (d) Radial-Shrink (PSNR = 30.31).

Table 1. Average PSNR values of denoising image over five runs for lena image.

\begin{tabular}{|c|c|c|c|c|c|}
\hline \multirow{2}{*}{ Denoising Algorithms } & \multicolumn{5}{|c|}{ Noise Standard Deviation } \\
\hline & 5 & 10 & 20 & 30 & 40 \\
\hline \multicolumn{6}{|l|}{ Decimated DWT } \\
\hline MMSE_TriShrink_Laplace [3] & 37.45 & 33.77 & 30.15 & 28.16 & 26.91 \\
\hline Radial-Shrink & 37.60 & 33.95 & 30.31 & 28.32 & 26.98 \\
\hline \multicolumn{6}{|l|}{ Redundant Wavelet Transform } \\
\hline Radial-Shrink (DT-CWT) & $38.17 *$ & $35.07 *$ & $31.51 *$ & $29.30 *$ & $28.04 *$ \\
\hline
\end{tabular}


Table 2. Average PSNR values of denoising image over five runs for boat image.

\begin{tabular}{|c|c|c|c|c|c|}
\hline \multirow{2}{*}{ Denoising Algorithms } & \multicolumn{5}{|c|}{ Noise Standard Deviation } \\
\hline & 5 & 10 & 20 & 30 & 40 \\
\hline \multicolumn{6}{|l|}{ Decimated DWT } \\
\hline MMSE_TriShrink_Laplace [3] & 35.94 & 32.32 & 28.75 & 26.73 & 25.31 \\
\hline Radial-Shrink & 35.96 & 32.43 & 28.89 & 26.82 & 25.46 \\
\hline \multicolumn{6}{|l|}{ Redundant Wavelet Transform } \\
\hline Radial-Shrink (DT-CWT) & $35.71 *$ & $33.08^{*}$ & $29.93 *$ & $27.95^{*}$ & $26.38 *$ \\
\hline
\end{tabular}

Table 3. Average PSNR values of denoising image over five runs for cameraman image.

\begin{tabular}{|c|c|c|c|c|c|}
\hline \multirow{2}{*}{ Denoising Algorithms } & \multicolumn{5}{|c|}{ Noise Standard Deviation } \\
\hline & 5 & 10 & 20 & 30 & 40 \\
\hline \multicolumn{6}{|l|}{ Decimated DWT } \\
\hline MMSE_TriShrink_Laplace [3] & 36.79 & 32.18 & 27.93 & 25.71 & 24.25 \\
\hline Radial-Shrink & 36.88 & 32.17 & 27.35 & 25.07 & 23.66 \\
\hline \multicolumn{6}{|l|}{ Redundant Wavelet Transform } \\
\hline Radial-Shrink (DT-CWT) & $37.09^{*}$ & $32.80^{*}$ & $28.47^{*}$ & $26.03^{*}$ & $24.64 *$ \\
\hline
\end{tabular}

\section{Discussion and Conclusion}

In this paper, we present a new image denoising algorithm based on Radial Exponential random vectors with local variance for modeling of wavelet coefficients in each subband, namely Radial-Shrink Instead of this density model other density models can be used. For example, instead of using Radial Exponential random vectors we can use a mixture model of this distribution. The performance of proposed technique is fairly good in terms of PSNR.

\section{References}

[1] Y. Zhou, S. Lai, L. Liu, and P. Lv, “An Improved Approach to Threshold Function De-noising of Mobile Image in CL2 Multi-wavelet Transform Domain," IEEE Signal Processing, 2000.

[2] L. Sendur and I. W. Selesnick, "Bivariate shrinkage functions for wavelet-based denoising exploiting interscale dependency," IEEE Transaction Signal Processing, Vol. 50, No. 11, pp. 2744-2756, November 2002.

[3] I. W. Selesnick "Estimation of Laplace Random Vectors in Adaptive White Gaussian Noise," IEEE Transactions on Signal Processing, Vol. 56, No. 8, pp. 3482-3496. August 2008.

[4] J. Portilla, V. Strela, M. Wainwright and E. P. Simon- celli, "Image denoising using scale mixtures of Gaussian in wavelet domain," IEEE Transaction Image Processing, Vol. 12, No. 11, pp. 1338-1351, November 2003.

[5] N. G. Kingsbury, "Image processing with complex wavelet," Phil. Transaction London A, September 1999.

[6] N. G. Kingsbury, "Complex wavelets for shift invariant analysis and filtering of signals," Applied Computation, Harmon, pp. 243-253., May 2001.

[7] S. M. M. Rahman, M. O. Ahmad, and M. N. S. Swamy, "Bayesian wavelet-based image denoising using the Gauss-Hermite expansion," IEEE Transaction Image Processing, Vol. 17, No. 10, pp. 1755-1771, October 2008.

[8] H. Rabbani, M. Vafadust, G. Saeed and I. W. Selesnick. "Image denoising employing a bivariate Cauchy distribution with local variance in complex wavelet domain," IEEE Signal Processing, Vol. 9, pp. 203-208, 2006.

[9] M. A. Chaudhry and S. M. Zubair, "Generalized incomplete gamma functions with application," Journal of Computer Applied Mathematic, Vol. 55, No. 1, pp. 99- 124, 1994.

[10] M. A. Chaudhry and S. M. Zubair, "On a class of incomplete gamma functions with applications," Chapman\& Hall, New York, 2001.

[11] D. L. Donoho and I. M. Johnstone, "Ideal spatial adaptation by wavelet shrinkage," Biometrika, Vol. 81, No. 3, pp. 425-455, 1994. 
[12] S. C. Choi and R. Wette, "Maximum likelihood estimation of the parameters of the gamma distribution and their bias," Technometric, Vol. 11, No. 4, pp. 683-690, 1969.

[13] M. K. Mihcak, I. Kozintsev, K. Ramchandran and P. Moulin, "Low-complexity image denoising based on statistical modeling of wavelet coefficients." IEEE Signal
Processing Letters, Vol. 6, No. 12, pp. 300-303, Decem-ber 1999.

[14] R. W. D. Nickalls, “A new approach to solving the cubic: Cardano's solution revealed," The Math-ematical Gazette, Vol. 77, pp. 354-359, 1993. 